\title{
Trypanosomosis of The Dromedary Camel (Camelus Dromedarius) and its Vectors in The Tsetse-free Arid Zone of North Eastern, Nigeria
}

\author{
MBAYA $^{1^{*}}$, A.W., IBRAHIM ${ }^{2}$, U.I. and APAGU ${ }^{1,}$ S.T.
}

${ }^{1}$ Department of Veterinary Microbiology and Parasitology, ${ }^{2}$ Department of Veterinary Medicine, University of Maiduguri, P.M.B. 1069, Maiduguri, Nigeria *Correspondence: email:_awmbaya@yahoo.com, Tel: +234 08036011774

\section{SUMMARY}

A cross sectional study on trypanosomosis of the dromedary camel (Camelus dromadarius) and its vectors in the tsetse free zone of northeastern Nigeria was undertaken. Out of 410 camels examined during the 12 month study period, 115 were infected. This was made up of $94(22.42 \%)$ males and $21(5.12 \%)$ females. This difference was significant $(\mathbb{P}<0.05)$. Similarly, infection was significantly higher $(P<0.05)$ in $80(19.51 \%)$ young camels ( $<4$ years) than $35(8.54 \%$ ) adults ( $>4$ years old). A mixed infection of Trypanosoma evansi $95(82.60 \%)$, Trypanosoma vivax $10(8.70 \%)$ and Trypanosoma congolense $10(8.70 \%$ ) were encountered. In decreasing order of sensitivity, the buffy coat technique detected $60(14.63 \%)$, thin blood smears $30(7.32 \%$ ), wet mount $20(4.88 \%)$ and thick blood smears $5(1.22 \%)$, the difference being significant $(P<0.05)$. Out of the 4,600 haematophagus arthropods vectors (Tabanus, Stomoxys, Hippobosca, Lyperosia) species caught in the area, blue biconical and blue NITSE traps baited with octenol (1-octen-3-0l), phenol (4methyl phenol) and ox-urine significantly $(p<0.05)$ caught more arthropod vectors than similarly baited black/grey biconical and black/grey NITSE traps. From the foregoing, the results showed that mixed trypanosome infections occur commonly among camels in the arid zone of northeastern Nigeria. Secondly, haematophagus arthropods vectors may be involved in the transmission process.

KEY WORDS: Trypanosomosis, Camel, Vectors, Arid zone, Nigeria

\section{Introduction}

Animal trypanosomosis constitutes a major threat to food security in several parts of sub-Saharan Africa including, Nigeria
Nigeria despite decades of chemotherapeutic control (Swallow, 2000). In endemic parts of Africa, the cyclical vector (Glossina) is responsible for transmitting Trypanosoma vivax, Trypanosoma congolense and Trypanosoma brucei. However, other haematophagus vectors such as Tabanus, Hippobosca, Lyperosia species and Stomoxys calcitrans have been incriminated in the mechanical (non-cyclical) transmission of Trypanosoma vivax, and Trypanosoma evansi among camels in tsetse free areas of the world (Mahmoud and Gray, 1980; Njiru et al., 2001). Similar non-cyclical transmissions have been reported as the method of maintaining the infection among cattle in the arid-northeastern, Nigeria (Mbaya, 1988; Nawathe et al., 1990; Nawathe et al., 1994). Camels are important source of meat, milk, transportation and draught power as well as by - products (wool, hair, skin and hides) in the arid zone of Northeastern Nigeria. Decreased productivity and mortality due to Trypanosoma evansi infection (surra) in camels have been described under natural and experimental conditions in other parts of the world (Mochoba et al., 2006; Ul Hassan et al., 2006; Dia, 2006).

In spite of the fact that the arid-zone of northeastern, Nigeria holds the highest concentration of the dromedary camel (Camelus dromedarius), there is a paucity of information regarding trypanosomosis in camels in the area. This study was therefore, designed to examine the prevalence of trypanosomosis among dromedary camels (Camelus dromedarius) and their vectors in 
the arid-zone of Northeastern Nigeria.

Materials and Methods

Study Area

The study was conducted in and around Maiduguri, the capital and largest urban center in Borno state, Nigeria. The state lies between latitude $11^{\circ} 05^{\prime} \mathrm{N}$ and $11^{\circ} 40^{\prime} \mathrm{N}$ and longitude $13^{\circ} 05^{\prime} \mathrm{E}$ and $13^{\circ} 25^{\prime} \mathrm{E}$. This area consists of a transition zone between the true dessert (Sahara) and the Sahel with annual rainfall below $200 \mathrm{~mm}$. The specific sampling sites were; Maiduguri cattle Market, Maiduguri Abattoir ante-mortem enclosure and 10 camel herds located at various areas within the arid-zone.

Parasitological techniques Blood samples with anticoagulant; ethylene diamine tetra acetate (EDTA) was collected from 410 young and old camels of both sexes via the jugular vein after the owners properly restrained them. The initial detection of motile forms of trypanosomes was by wet mount. Thin, thick and buffy coat smears were stained with $10 \%$ Giemsa stain and examined for trypanosomes (Murray et al., 1983). Morphological identification of trypanosomes was by standard criteria with either stained buffy-coat smears or thin smears (Soulsby, 1982; Paris et al., 1982). Blue and black/grey biconical, as well as blue and black/grey NITSE traps baited with octenol (1-octen-3-ol), phenol (4-methyl phenol) and ox-urine were used to capture vectors at the various sampling sites. The 89 traps were placed at a spacing of 25 meters according to the methods of Siziya, (1994) to trap haematophagus arthropod vectors throughout the period of the study, while morphological identification of the vectors was carried out using standard keys (Soulsby, 1982).

\section{Statistical Analysis}

Data collected were, subjected to t-test in pair wise comparison of prevalence rates where values less than 0.05 were, considered significant. A 2 × 2 contingency table was used for analysis of relative risk, Chi-square for independence and trend to give a measure of strength of association between the variables (Maed and Curnow, 1983).

\section{Results}

The prevalence of trypanosomosis in the one humped camel according to sex and age is presented in Table I. Out of the 410 camels examined, 280 were males and 130 females. From this $94(22.42 \%)$ of the males and $21(5.12 \%)$ of the females were infected. The males had statistically higher $(p<0.05)$ prevalence of infection than the females. Similarly, the young camels ( $<4$ years old), had a higher $(\mathrm{p}<0.05)$ prevalence of $80(19.51 \%)$ as compared to $35(8.54 \%)$

among the adults ( $>4$ years old).

Table II shows a mixed infection with various trypanosome species encountered among the 115 infected camels. Trypanosoma evansi occurred more commonly $(\mathrm{p}<0.05)$ in $95(82.60 \%)$ of the camels, followed by Trypanosoma vivax and Trypanosoma congolense, which, both equally had $10(8.70 \%)$ prevalence rates Meanwhile, no other haemoparasites were seen among the camels during the study.

TABLE I: Prevalence of trypanosomosis of the dromedary camel (Camelus dromedarius) examined in the arid zone of Northeastern, Nigeria according to sex and age

\begin{tabular}{|llcc|}
\hline Parameters & & No. Examined & No. Infected (\%) \\
Sex & Male & 280 & $94(22.42) \mathrm{a}$ \\
& Female & 130 & $21(5.12) \mathrm{b}$ \\
& Total & 410 & $115(28.05)$ \\
Age & Young (< 4years) & 115 & $80(19.51) \mathrm{a}$ \\
& Adults (> 4 years) & 295 & $35(8.54) \mathrm{b}$ \\
& Total & 410 & $115(28.05)$ \\
\hline
\end{tabular}


TABLE II: Trypanosome species detected in blood of dromedary camels (Camelus dromedaries) in the arid zone, Northeastern, Nigeria

\begin{tabular}{|cll|}
\hline No. Examined & No. Infected (\%) & Trypanosome species encountered (\%) \\
\hline 410 & $115(28.05)$ & (i) Trypanosoma evansi 95(82.60)a \\
& & (ii) Trypanosoma vivax 10(8.70)b \\
& (iii) Trypanosoma congolense 10(8.70)c \\
\hline
\end{tabular}

Superscripted values $(a, b, c)$ in column differed significantly, $(p<0.05)$.

Table III shows the different diagnostic techniques employed in detecting the infection among the camels. In decreasing order of sensitivity, the buffy coat technique (BMC), detected 60(14.63\%), followed by thin smears $30(7.32 \%)$, wet mount $20(4.88 \%)$, then thick smears, which detected only 5(1.22\%). Table IV shows the various mechanical arthropod vectors caught near various locations where the camels were, sampled over the 12-month period. A total of 4, 600 haematophagus arthropod vectors were caught using the various traps. The species of vectors caught were; Tabanus, Hippobosca, Lyperosia species and Stomoxys calcitrans. Tabanus species was the most predominantly encountered of the four species in that order. Table V summarizes the efficacy of the various types of traps used in the study. The blue biconical and blue NITSE traps baited with olfactory attractants such as octenol (1octenol-3-ol), phenol (4-methyl phenol) and ox-urine, significantly $(\mathrm{p}<0.05)$ caught more mechanical arthropod vectors than similarly baited black/ grey biconical and black/ grey NITSE traps.

TABLE III: Sensitivity of the various parasitological techniques used in the etection of trypanosomes

\begin{tabular}{|ll|}
\hline Parasitological techniques & No. positive (\%) \\
\hline Buffy coat examination & $60(14.63) \mathrm{a}$ \\
Thin blood smear & $30(7.32) \mathrm{b}$ \\
Wet mount & $20(4.88) \mathrm{c}$ \\
Thick blood smears & $5(1.22) \mathrm{d}$ \\
Total & $115(28.05)$ \\
\hline
\end{tabular}

Superscripted values a, b, c, d differed significantly $(p<0.05)$.

TABLE IV: Haematophagus arthropod vectors and other flies caught at various camel locations in the arid zone of northeastern, Nigeria during the study

\begin{tabular}{|llll|}
\hline Locations & Total catch & Vectors encountered & Number (\%) \\
\hline $\begin{array}{l}\text { Maiduguri } \\
\text { cattle market }\end{array}$ & \multirow{2}{*}{800} & (i) Tabanus spp. & $1000(55.56) \mathrm{a}$ \\
& & (ii) Stomoxys calcitrans & $700(38.89) \mathrm{c}$ \\
& & (iii) Hippobosca spp. & $60(3.33) \mathrm{d}$ \\
& & (iv) Lyperosia spp. & $30(1.67) \mathrm{e}$ \\
(v) Musca domestica & $10(0.56) \mathrm{e}$ \\
$\begin{array}{l}\text { Abattoir } \\
\text { ante mortem }\end{array}$ & \multirow{2}{*}{850} & (i) Tabanus spp. & $700(82.24) \mathrm{b}$ \\
enclosure & & (ii) Stomoxys calcitrans & $100(11.76) \mathrm{f}$ \\
& & (iii) Calliphora spp. & $50(5.88) \mathrm{d}$ \\
\hline
\end{tabular}




\begin{tabular}{|llll|}
\hline Locations & Total catch & Vectors encountered & Number (\%) \\
\hline Camel herds & \multirow{2}{*}{$(\mathrm{n}=1050$} & (i) Tabanus spp. & $1,600(82.5) \mathrm{b}$ \\
& & (ii) Stomoxys calcitrans & $200(10.26) \mathrm{f}$ \\
& & (iii) Hippobosca spp. & $100(5.13) \mathrm{d}$ \\
& & (iv) Lyperosia spp. & $25(1.28) \mathrm{e}$ \\
& & (v) Musca domestica & $25(1.28) \mathrm{e}$ \\
\hline \hline
\end{tabular}

Superscripted values (a, b, c, d, e, f) differed significantly $(p<0.05)$. $(n=10)=10$ different herds sampled within the arid zone

TABLE V: The effect of visual attraction on baited biconical and NITSE traps on the population dynamics of haematophagus arthropod vectors caught in or around camel habitats in the arid zone of Northeastern, Nigeria

\begin{tabular}{llll} 
Type of traps & Visual attraction & No. of traps used & Total catch \pm S.D. \\
\hline Biconical & Blue & 20 & $3,000 \pm 0.44 \mathrm{a}$ \\
& Black/grey & 26 & $600 \pm 0.30 \mathrm{~b}$ \\
& Total & 46 & 3,600 \\
NITSE & Blue & 23 & $200 \pm 0.14 \mathrm{c}$ \\
& Black/grey & 20 & $800 \pm 0.34 \mathrm{~d}$ \\
& Total & 43 & 1,000
\end{tabular}

Superscripted values ${ }^{a, b, c, d}$ differed significantly $(p<0.05)$. All traps were baited with olfactory attractants (octenol, phenol and ox-urine).

\section{DISCUSIONS}

The overall prevalence of $28.05 \%$ in this study is higher than $2.5 \%$ previously reported in the same area, almost two decades ago (Egbe-Nwiyi and Chaudry, 1994) or $6.5 \%$ in the Sudan Savannah region of Nigeria (Falope, 1986). This is probably due to the recent influx of livestock into the sanitary cordon of the arid zone of North-eastern Nigeria from neighboring Republics of Chad, Niger, and Southern Sudan due to civil unrest in some of the areas (Chardonnet and Maillard, 2002). Livestock resources survey conducted by Bourn et al. (1994) indicated that over 13.9 million of the total livestock populations in the tsetse free arid-zone of northeastern Nigeria are situated in both wet and dry seasons especially on the fringes of the Lake Chad. However, most recently, the effects of desertification, prolonged dry season, low rainfall and the upstream damming of the major tributaries of the Lake, has made the area unfavorable for livestock than in the previous years. In view of this, seasonal migration by most pastoralists with their camels as the main source of transportation or draft power into tsetse-infested areas might have increased their risk of infection, hence the likely factor responsible for the higher prevalence with a mixed infection of Trypanosoma evansi, Trypanosoma vivax and Trypanosoma congolense. The significance of this finding among camels in the arid zone of northeastern Nigeria is that, mixed infection of trypanosomes is being reported for the first time after a previously reported single Trypanosoma evansi infection (Egbe-Nwiyi and Chaudry, 1994). In spite of the mixed infection, however, Trypanosoma evansi was the most predominant of the trypanosome species encountered. It has been identified as the principal cause of camel trypanosomosis (Surra) by several researchers (Falope, 1986; Egbe-Nwiyi and Chaudry, 1994; Mochoba et al., 2006; Ul Hassan et al., 2006; Dia, 2006). Similarly, mixed infections of 
Trypanosoma congolense, Trypanosoma brucei and Trypanosoma vivax have been reported from camels in Sudan. Their role in camel trypanosomosis is insignificant. However, the infected camels may serve as a major source of infection for other livestock in an area (Elamine et al., 1999).

Tsetse flies (Glossina) have been reported to have a flight range of $15 \mathrm{~km}$ from their habitat and beyond this, transmission of trypanosomes occur mechanically (Nawathe et al., 1994). The results of this study, agrees with the fact that mechanical transmission by Tabanus, Stomoxys, Hippobosca and Lyperosia species is likely the mode of transmission in the arid zone in the absence of Glossina. Mechanical transmission in tsetse free zones of Africa, Asia and America is also due to similar vectors (Soulsby, 1994). The efficacy of the blue biconical and blue NITSE traps over the black/ grey biconical and black/grey NITSE traps baited with olfactory attractants might be associated with the visual affinity of the haematophagus arthropod vectors to the colour blue. The colour blue as well as olfactory attractants enhance trap catches (Phelps and Holloway, 1992; Amsler et al., 1994; Mihok, 1995; Mihok and Carlson, 2007).

Age also played a significant role in the prevalence of infection. Young camels of less than 4 years had higher prevalence of infection than the adult camels over 4 years of age. This might be due to age susceptibility and lack of premunity among the young (Soulsby, 1982). Higher prevalence was also observed among the

\section{REFERENCES}

AMSLER, S., FILLEDIER, J. and MILLIGAN, R. (1994): Attractiveness for Tabanidae of various tsetse traps with or without olfactory attractants. Preliminary results obtained in Burkina Faso. Rev. Elev. Méd. Vét. Pays trop., 47, 63-68.

BOURN, D., WINT, W., BIEUCH, R. and WOOLLEY, E. (1994): Nigerian livestock resources survey. Wld Anim. Rev., 78, 49 58. males than in the females, which might likely be associated with roaming from one herd to another when, the females are on heat, thereby increasing their chances of contact with the vectors. Among the conventional parasitological techniques used for the detection of the trypanosome infections in the camels, buffy coat examination either as direct examination of the buffy coat or staining its wet film with 10\% Giemsa stain gave the best result. This is probably, because it is the only concentration technique used in the study. This method was also most efficient in detecting the first outbreak of Trypanosoma vivax infection of cattle in the tsetse free arid zone of northeastern Nigeria (Mbaya, 1988; Nawathe et al., 1994).

\section{CONCLUSION}

In conclusion, mixed infection of trypanosomes due to T. evansi, T. vivax and T. congolense in camel is being reported for the first time in the tsetse-free arid zone of northeastern Nigeria. Similarly, the prevalence of infection was equally high. It is therefore, recommended that chemoprophylaxis, chemotherapy and vector control be put in place in order to reduce the effect of trypanosomosis in the dromedary camel in the region.

\section{ACKNOWLEDGEMENTS}

The efforts of the staff and management of Borno State Ministry of Agriculture and Maiduguri Abattoir is highly appreciated. The provision of some literature by Professor Egbe-Nwiyi of the Department of Pathology, University of Maiduguri, is also appreciated.

CHARDONNET, B. and MAILLARD, A. (2002): Wildlife epidiomiosurveillance. PACE Training manual, Yankari National Park; 23-24.

DIA, M.L. (2006): Parasites of the camel in Burkina Faso, Trop. Anim. Hlth. Prod., 38, 17-21.

ELAMINE, E.A., ELBASHIR, M.O.A. and SAHEED, E.M.A. (1999): Prevalence and infection pattern of Trypanosoma evansi in camels in Middle Eastern Sudan. $\mathrm{Tr}$ o $p$. 
Anim. Hlth Prod., 30, 107-114.

EGBE-NWIYI, T.N. and CHAUDRY, S.U.R. (1994): Trypanosomosis; Prevalence and pathology of camel of arid zone of Northeastern Nigeria, Pakistan Vet. J., 14, $24-27$

FALOPE, O.O. (1986): The prevalence of trypanosomiasis in camels (Camelus dromedarius) in Kaduna town Nigeria, (M.P.V.M. dissertation, University of Ibadan; 1-6.

MAED, R. and CURNOW, R.N. (1983). Statistical methods in Agriculture and Experimental biology. Chapman and Hall, London; 3-9.

MAHMOUD, M.F. and GRAY, A.R. (1980): Trypanosomosis due to Trypanosoma evansi: A review of recent research. Trop. Anim. Hlth. Prod., 12, 35-47.

MBAYA, A.W. (1988): Survey of Trypanosoma vivax infection of cattle in the arid zone. DVM dissertation, University of Maiduguri, Nigeria; 34-56.

MIHOK, S. (1995): Trapping technique for Stomoxys species, In: Organization of African Unity / Scientific, Technical Research Commission report, 18, 268 210.

MIHOK, S.A. and CARLSON, D.A. (2007): Performance of painted plywood and cloth Nzi traps relative to Manitobia and green head traps for Tabanidae and stable flies. J. Econ. Entomol., 100, 613-618.

MOCHOBA, M.O., KITAL, P.M., GATHURA, P.B., OGARA, W.D., EREGAE, F.M., KAITHO, T.D. and CATLEY, A. (2006): The socio-economic impact of important camel diseases as perceived by a pastoralist community in Kenya. Ond. $J$. Vet. Res., 73, 269 - 274

MURRAY, M., TRAIL, J.C.M., TURNER, D.A. and WISSOQ, Y. (1983): Livestock productivity and trypanotolerance. ILCA Network training manual, Addis Ababa, Ethiopia; 67-68.

NAWATHE, D.R., SINHA, P.K. and ABECHI, A.S. (1990): Acute bovine trypanosomosis in the arid zone. In:
Proceedings of the $27^{\text {th }}$ Annual Conference of the Nigerian Veterinary Association, Maiduguri; $1-4$.

NAWATHE, D.R., SRIVASTAVA, G.C. and SINHA, P.K. (1994): Survey of animal trypanosomosis and its factors in the arid zone of Borno State. Uni. Maid. Occas. Publ., 2, 1-5.

NJIRU, Z.K., OLE-MAPENY, I.M., OUMA, J.O., NDUNGU, J.M., and OLAHO- MUKANI, W. (2001): Prevalence of trypanosomosis in camel calves: A pilot study in Laikipia district of Kenya. Rev. Elav. Med. Pays Trop., 34, 183-186.

PARIS, J., MURRAY, M. and MCODIMBA, F. (1982): A comparative evaluation of parasitological techniques currently available for the diagnosis of African trypanosomiasis in cattle, Acta Trop., 39, $301-316$

PHELPS, R.J. and HOLLOWAY, M.T.P. (1992): Catches of Tabanidae in response to visual and odour attractants in Zimbabwe. J. Afr. Zool., 106, 371 - 380.

SIZIYA, M. (1994): Movement and distribution of tsetse flies in Nguruman, South Western Kenya. PhD thesis, University of Zambia; 23-45.

SOULSBY, E.J.L. (1982). Helminthes,

Arthropods and Protozoa parasites of domesticated Animals, Bailere Tindal, London; 232-233.

SOULSBY, E.J.L. (1994): How parasites tolerate their hosts. Brit. Vet. J., 8, 150 312.

SWALLOW, B.M. (2000): Impacts of trypanosomosis in African agriculture. Programme against African Trypanosomosis Technical and Scientific series, F. A. O., 2, 45-46.

UL HASSAN, M., MUHAMMAD, S., GULIERREZ, C.,IQBAL, Z., SHAKOUR, A. and JABBARI, A. (2006): Prevalence of Trypanosoma evansi infection in equines and camels in the Punjab region of Pakistan. Ann. New York Acad. Sci., $1081,322-324$. 